\title{
Dynamic Equivalencing Supported by Load Disaggregation via Harmonic Current Analysis
}

\author{
Georgios A. Barzegkar-Ntovom (iD \\ Theofilos A. Papadopoulos \\ Athanasios S. Pavlakis \\ Dept. of Electr. \& Comput. Eng. \\ Democritus University of Thrace \\ Xanthi, Greece \\ gbarzegk@ee.duth.gr \\ thpapad@ee.duth.gr \\ athapav11@ee.duth.gr
}

\author{
Eleftherios O. Kontis \\ Dept. of Electr. \& Comput. Eng. \\ Aristotle University of Thessaloniki \\ Thessaloniki, Greece \\ ekontis@auth.gr
}

\author{
Mazheruddin H. Syed \\ Efren Guillo-Sansano \\ Graeme M. Burt \\ Institute for Energy and Environment \\ University of Strathclyde \\ Glasgow, UK \\ mazheruddin.syed@strath.ac.uk \\ efren.guillo-sansano@strath.ac.uk \\ graeme.burt@strath.ac.uk
}

\begin{abstract}
The time-varying composition of power system loads influences the robustness of measurement-based dynamic equivalent models, constituting them valid only for specific operating conditions. To overcome this issue, in this paper, a new generic dynamic equivalencing method, utilizing load disaggregation, is proposed. Load disaggregation by analyzing the aggregated harmonic current content is used to estimate the load composition of a distribution network, providing higher visibility and information regarding its operational condition. In this sense, representative sets of dynamic load model parameters are obtained for different loading conditions using a clustering algorithm. The accuracy and robustness of the proposed method is assessed using laboratory measurements.
\end{abstract}

Index Terms-Clustering, dynamic modelling, harmonic analysis, load disaggregation, measurement-based approach

\section{INTRODUCTION}

Accurate load models are essential for the analysis and secure power system operation and control [1]-[3]. Several studies have indicated the importance of accurate load representation, especially regarding voltage and frequency stability assessment [4], [5].

To derive aggregated equivalent models, the component- and the measurement-based approaches are used [1]. The former is a bottom-up modelling method that requires a priori knowledge of the examined grid. Despite its distinct advantages, this approach cannot be easily applied since information regarding the load composition is not always available to power system operators [1]. In the second one, equivalent models are derived using measurements collected from representative substations and feeders [1].

In the measurement-based method, model parameters are identified by fitting the measured data to a predefined load

Laboratory measurements were conducted in the frame of ERIGrid project "Enhanced Generic Load Modelling using Harmonic Profiles (HaRMoNic)" with Grant Agreement No. 654113.

The research work of G. A. Barzegkar-Ntovom was supported by the Hellenic Foundation for Research and Innovation (HFRI) under the HFRI PhD Fellowship grant (Fellowship Number: 1318). model structure using parameter estimation techniques [4]. A significant advantage of this approach is that load dynamics are directly captured [6], resulting in accurate load models, since data from the actual network are used. However, in most cases, the derived models are valid only for a given operating condition, while the estimation procedure must be repeated for every new operating condition [7], [8]. Hence, model parameters estimated using disturbance data at a specific power system location may not be applicable to simulate adequately different cases, e.g., network configurations, operating conditions, etc. [9].

To extend the applicability of the equivalent models to simulate accurately a wide range of distinct network conditions, generalization techniques must be examined. In literature, several approaches have been proposed to develop generic equivalent models. For example, in [7] and [10], statistical analysis is applied to identified model parameters for the derivation of generic equivalent models. In [11] generic parameters are obtained using artificial intelligence techniques. In [9] clustering and artificial neural networks are combined to derive representative sets of parameters for a variable-order dynamic equivalent model. Finally, in [12], different clustering methods are examined to classify identified parameters for the development of generic models.

Scope of this paper is to propose a new methodology for the development of generic measurement-based equivalent models for the dynamic analysis of power systems over a wide range of operational conditions. The main advantage of the proposed method relies on the use of load disaggregation based on harmonic current analysis. To the best of the authors' knowledge, load harmonic disaggregation to enhance the development of generic dynamic load models is examined for the first-time in the archived literature. In particular, load disaggregation is applied to estimate the fractions (participation) [13] of specific load classes into the total load demand; higher visibility is achieved regarding the load composition of the examined network. Using this information as input, representative sets of 
dynamic load model parameters are obtained on the basis of cluster analysis, corresponding to a wide range of operational conditions. For the generic load equivalents, the widely-used exponential recovery model (ERM) structure [14] is adopted. Following classification into different groups, system operators can employ the corresponding sets of model parameters to simulate unseen disturbances, if load composition is known through harmonic disaggregation. The effectiveness of the proposed method is evaluated and validated under different network configurations and voltage disturbances, using measurements obtained in a laboratory-scale distribution system.

\section{Proposed Methodology}

The process to derive the proposed generic dynamic equivalent model is summarized in Fig. 1. A database of $M$ data sets is developed, using measurements recorded at the point of common coupling (PCC), e.g., secondary side of the substation medium-voltage transformer. Each data set contains the harmonic content of the aggregated load under steadystate operation, as well as the bus voltage, real and reactive power dynamic responses when an event occurs, e.g., a voltage disturbance. In this paper, the harmonic content is used to decompose the aggregated load demand into specific load classes, i.e., static load (SL), converter type (Conv), motors (MTR) and non-linear loads (NLL), each with given current harmonic and dynamic characteristics. For this purpose, current harmonic disaggregation is applied and the fractions of the load classes are determined. The mean values of amplitude and phase angle data over a long enough period of recording time (in the order of minutes) are used as the representative values for the harmonic content of the load. In addition, the bus voltage, real and reactive power dynamic responses are used to analyze the network dynamics, by developing the corresponding dynamic equivalent model. In this work, dynamic equivalents are derived using the ERM. To develop generic equivalent models (training procedure), a subset $\left(M_{T D}\right)$ of the total $M$ data sets are used. The calculated load class fractions and the corresponding ERM parameters are introduced as

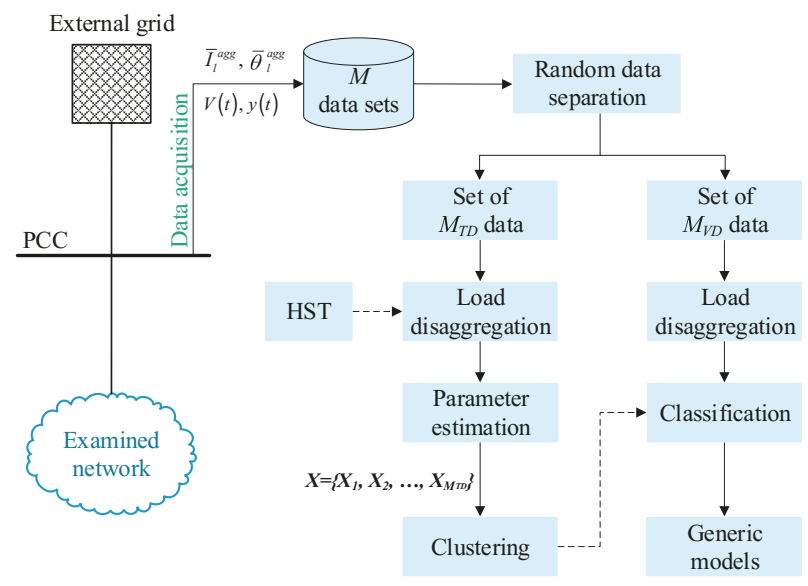

Fig. 1. Procedure used to develop generic equivalent models. inputs into a clustering algorithm to group the available $M_{T D}$ data into representative sets of distinct operational conditions.

The derived robust set of parameters is used to validate the performance of the developed equivalents for the remaining $M_{V D}$ data sets. This procedure is also applied to derive dynamic equivalent model parameters for future operational conditions not contained in the original $M$ data sets. Considering a new operational condition the most suitable representative set of parameters is selected in terms of a classification algorithm.

\section{A. Harmonic Signature Table}

The adopted disaggregation procedure is based on identifying the minimum difference between the harmonic current vector of the measured aggregate harmonic load content and the vector sum of the harmonic current spectrum of each load class [13], [15]. In order to investigate a wide range of operational conditions, the use of the harmonic signature table (HST) concept is introduced as a preparatory step of the proposed procedure; HST includes $H$ possible vector sums of the harmonic current spectrum of the given load classes, assuming different network configurations (load mix) and loading conditions [16]. The characteristic harmonic current spectrum patterns for each load class can be obtained offline by conducting laboratory measurements or from available relevant published data [13].

\section{B. Load Disaggregation}

To determine the components that form an aggregated load, harmonic measurements acquired at the PCC under steadystate operation are used. Load disaggregation is performed by finding the minimum difference between the harmonic content of the aggregate load and the harmonic vector sums included in the HST. This is achieved by simultaneously comparing the corresponding current harmonics (odd-order harmonic measurements from the fundamental to the $7^{\text {th }}$ ) as shown in (1)

$$
\underset{h \in H}{\operatorname{argmin}} \sum_{l}\left(\left|\bar{I}_{l}^{a g g} \cdot e^{j \bar{\theta}_{l}^{a g g}}-\right| \bar{I}_{l h}^{H S T}\left|\cdot e^{j \bar{\theta}_{l h}^{H S T}}\right|\right)
$$

where, $\bar{I}_{l}^{a g g}$ and $\bar{\theta}_{l}^{a g g}$ are the mean amplitude and phase angle values of the $l$-th $(l=1,3,5,7)$ harmonic current of the aggregated load, respectively; $\bar{I}_{l h}^{H S T}$ and $\bar{\theta}_{l h}^{H S T}$ are the mean amplitude and phase angle values of the l-th harmonic current for the $h$-th $(h=1, \ldots, H)$ possible permutation of the HST, respectively. Minimizing (1) leads to the identification of the load mix that comprises the network as well as the corresponding loading condition. This results into the vector containing the fraction of each load class into the total demand, denoted as $\boldsymbol{\alpha} ; \boldsymbol{\alpha}$ is defined in (2), where $m$ is the number of the assumed load classes. In this paper, $m$ is equal to 4 .

$$
\begin{gathered}
\boldsymbol{\alpha}=\left[\alpha_{1}, \alpha_{2}, \ldots, \alpha_{m}\right], \\
\sum_{i=1}^{m} \alpha_{i}=100 \%, \quad 0 \leq \alpha_{i} \leq 100 \%
\end{gathered}
$$




\section{Derivation of Indicative Parameters Using Clustering}

To model the measured dynamic power responses and to derive generic equivalents, the ERM is adopted. The mathematical representation of the ERM is given in (3) and (4)

$$
\begin{gathered}
y_{e}(t)=y_{s}(t)-\left(y_{s}(t)-y_{t}(t)\right) \cdot e^{-\frac{t-t_{0}}{T_{y}}} \\
y_{s}(t)=y_{0}\left(\frac{V(t)}{V_{0}}\right)^{N_{s}} \quad y_{t}(t)=y_{0}\left(\frac{V(t)}{V_{0}}\right)^{N_{t}}
\end{gathered}
$$

where, $y_{e}(t)$ is the estimated real and/or reactive power and $V(t)$ is the grid voltage; $t_{0}$ is the time instant of the disturbance and $y_{0}$ and $V_{0}$ denote the power demand and voltage magnitude prior to the disturbance. $T_{y}$ is the recovery time constant; $N_{s}$ and $N_{t}$ are the steady-state and transient voltage exponents, respectively. For real and reactive power modelling, the following sets of parameters must be identified: $\boldsymbol{\theta}_{\boldsymbol{P}}=$ $\left[N_{s, p}, N_{t, p}, T_{y, p}\right]$ and $\boldsymbol{\theta}_{\boldsymbol{Q}}=\left[N_{s, q}, N_{t, q}, T_{y, q}\right]$, respectively. $\boldsymbol{\theta}_{\boldsymbol{P}}$ and $\theta_{Q}$ are estimated using the nonlinear least square (NLS) optimization technique [1], targeting to minimize the objective function of (5)

$$
J=\sum_{n=1}^{N}\left(y[n]-y_{e}[n]\right)^{2}
$$

where $N$ is the total number of the response samples and $y[n]$, $y_{e}[n]$ are the measured and estimated, real or reactive power response at the $n$-th sample, respectively.

To automatically partition the available data sets into $K$ clusters that present similar characteristics, cluster analysis is applied. Clustering is performed by combining $\boldsymbol{\theta}_{\boldsymbol{P}}, \boldsymbol{\theta}_{\boldsymbol{Q}}$ and $\boldsymbol{\alpha}$ for each of the $M_{T D}$ data sets, into one single vector $\boldsymbol{X}_{\boldsymbol{j}}=\left[\boldsymbol{\theta}_{\boldsymbol{P}, \boldsymbol{j}}, \boldsymbol{\theta}_{\boldsymbol{Q}, \boldsymbol{j}}, \boldsymbol{\alpha}_{\boldsymbol{j}}\right]$ (here $j=1, \ldots, M_{T D}$ ). Thus, the input to the clustering algorithm is the set $\boldsymbol{X}$ of $M_{T D}$ observations $X=\left\{X_{1}, X_{2}, \ldots, X_{M_{T D}}\right\}$. To estimate $\boldsymbol{\theta}_{P, j}, \boldsymbol{\theta}_{Q, j}$, voltage, real and reactive power disturbance data recorded at the PCC are used. To estimate $\boldsymbol{\alpha}_{\boldsymbol{j}}$, disaggregation is applied to the corresponding harmonic content of the aggregate load, prior to each disturbance under consideration. In this work, the $\mathrm{k}$-medoids clustering approach is employed, where a set of observations is divided into $K$ clusters so that the subsets minimize the sum of distances between an observation and a center of the observation's cluster [17]; the center of the subset, namely medoid, is a member of the subset. To minimize the sum of distances from each observation to its cluster medoid, over all $K$ clusters, the partitioning around medoids (PAM) iterative algorithm is used [17]. The optimal number of clusters is determined using the knee-point criterion for the curve of the Within Cluster sum of squares to Between Cluster sum variation Ratio (WCBCR) [18]. Using this procedure, $K$ representative sets of real and reactive power model parameters $\Theta_{k}^{\prime}=\left\{\theta_{P, k}^{\prime}, \theta_{Q, k}^{\prime}\right\}$ are obtained for $\boldsymbol{\alpha}_{k}^{\prime}$ loading conditions (here $k=1, \ldots, K$ ).

\section{Application and Validation of the Proposed Method}

A major advantage of the proposed method is that it can be used for online applications. Once the training (i.e., derivation of model parameters, clustering, etc.) is completed, representative sets of model parameters are derived for distinct operating conditions; these sets can be used for online applications. Under steady-state operation, the load composition of the network, denoted as $\boldsymbol{\alpha}_{\boldsymbol{n} \boldsymbol{e}}$, is continuously calculated. This is achieved by decomposing the harmonic content of the aggregated load demand into specific load classes. Subsequently, the $\Theta_{k}^{\prime}$ that mostly represents $\boldsymbol{\alpha}_{n e t}$ is determined using a classification process. Specifically, the Euclidean distance between the vectors $\boldsymbol{\alpha}_{n e t}$ and $\boldsymbol{\alpha}_{\boldsymbol{k}}^{\prime}$ is computed; the resulting $k$-th set is determined by minimizing (6)

$$
\underset{k}{\operatorname{argmin}} \sum\left\|\boldsymbol{\alpha}_{n e t}-\boldsymbol{\alpha}_{\boldsymbol{k}}^{\prime}\right\|
$$

The accuracy of the proposed method is assessed using the $M_{V D}$ data sets. Specifically, the performance of the derived generic models is evaluated using the $M_{V D}$ data sets. For this reason, the relative error $(\epsilon)$ is calculated as a metric to evaluate the accuracy between the measured dynamic responses and the derived equivalents [19]; $\epsilon$ is defined as

$$
\epsilon=100 \% \cdot \frac{\left(\frac{1}{N} \sum_{n=1}^{N}\left(y[n]-y_{e}[n]\right)^{2}\right)^{\frac{1}{2}}}{\left(\frac{1}{N} \sum_{n=1}^{N} y[n]^{2}\right)^{\frac{1}{2}}} .
$$

Note that, a dynamic response is said to be adequately simulated, if $\epsilon$ is less than 5\% [19].

\section{SYSTEM UNDER STUDY}

\section{A. Network Configuration}

The effectiveness of the proposed methodology is investigated using measurements obtained in the three-phase, $400 \mathrm{~V}$, $50 \mathrm{~Hz}$, laboratory-scale grid as in Fig. 2. The test setup is supplied by a three-phase programmable voltage source (PVS) and consists of a SL bank rated at $40 \mathrm{~kW}$, a $8 \mathrm{~kW}$ back-to-back power converter unit, a $5.5 \mathrm{~kW}$ MTR representing the dynamic load part of the aggregate load and a $6 \mathrm{~kW}$ NLL composed of a full wave diode rectifier connected to an inductance of $16 \mathrm{mH}$ in series with a variable resistive load bank. The dynamic responses of the bus voltage, real and reactive power are

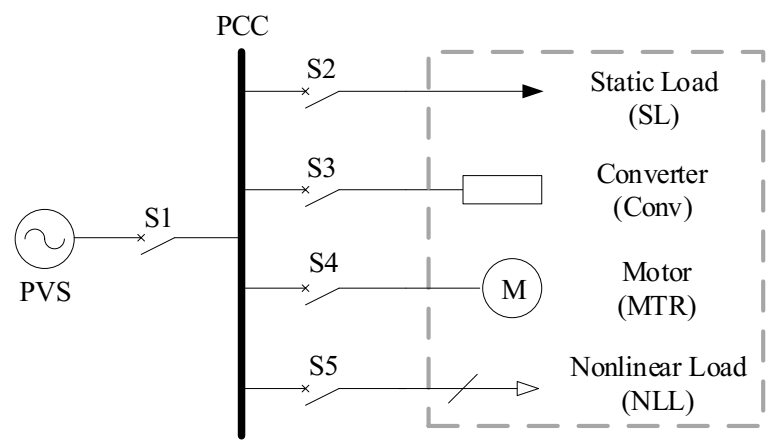

Fig. 2. Experimental setup. 
measured by applying an adaptive filter-based phasor measurement unit (PMU) algorithm, implemented in hardware [20]. To measure the harmonic components a similar algorithm was implemented based on the sliding Discrete Fourier Transform (DFT) algorithm [21].

\section{B. Test Cases}

Initially, odd-order harmonic current vectors, from the fundamental to the $7^{\text {th }}$ harmonic were recorded for each load class separately under different loading conditions; these data are used to generate the HST. The harmonic current spectra of each load class is shown in Table I. Specifically, the range of the harmonic components, i.e., $i_{l}(\%)$ (percentage of harmonics of order $l$ with respect to the fundamental), and the corresponding phase angles are presented. The harmonic current profiles were continuously recorded under steady-state operation for $5 \mathrm{~min}$ at a rate of 1 sample per second (sps). It is shown that mainly the NLL is dominated by $5^{\text {th }}$ and $7^{\text {th }}$ harmonic currents. Afterwards, harmonic current profiles were recorded for a total of $M_{c o m b}=18$ test cases (TCs) of aggregated load. The examined TCs correspond to a variety of network configurations and operational conditions implemented by switching on and off the corresponding switches, i.e., S2 - S5 (see Fig. 2), and by altering the power of the installed components; the examined TCs are summarized in Table II.

Additionally, for each TC, $M_{v}=20$ voltage disturbances, varying from -0.1 p.u. to 0.1 p.u., are introduced using the PVS. The resulting voltage, real and reactive power responses are recorded at a data rate of 100 sps. Hence, a set of $M_{\text {tot }}=M_{c o m b} \cdot M_{v}=360$ disturbances acquired during different network configurations, loading conditions and voltage events, are generated. The disturbances are randomly divided into two different groups; the training group contains $85 \%$ of the available data, i.e., $M_{T D}=306$, whereas the remaining sets, i.e. $M_{V D}=54$, were used to validate the efficiency of the derived generic models.

\section{Application of the PROPOSED METHODOLOGY}

In this Section, the proposed load disaggregation process to determine the load mix of the network is assessed. Moreover, the robustness of the derived generic models is validated.

TABLE I

HARMONIC CURRENT SPECTRA OF INDIVIDUAL CONNECTED LOADS*

\begin{tabular}{c|c|c|c|c|c}
\hline \hline \multicolumn{2}{l}{ Harmonic } & SL & MTR & Conv & NLL \\
\hline \multirow{2}{*}{ 3rd } & $i_{l}(\%)$ & $0.5-1.0$ & $0.3-0.9$ & $0.5-1.9$ & $0.3-0.4$ \\
& $\theta_{l}\left({ }^{\circ}\right)$ & $9.7-19.0$ & $98.8-129.6$ & $-36.5-(-30.8)$ & $-60.7-8.7$ \\
\hline \multirow{2}{*}{ 5th } & $i_{l}(\%)$ & $0.1-0.2$ & $0.5-1.6$ & $2.2-3.1$ & $22.0-22.4$ \\
& $\theta_{l}\left({ }^{\circ}\right)$ & $-37.1-(-27.2)$ & $-97.4-(-52.4)$ & $-26.9-(-1.2)$ & $63.7-72.9$ \\
\hline \multirow{2}{*}{ 7th } & $i_{l}(\%)$ & $0.2-0.3$ & $0.8-1.5$ & $1.4-2.0$ & $10.8-11.0$ \\
& $\theta_{l}\left({ }^{\circ}\right)$ & $-163.3-(-147.8)$ & $-92.3-(-56.0)$ & $-118.0-(-97.3)$ & $87.7-92.0$ \\
\hline \hline
\end{tabular}

*As resulted from the following examined loading conditions (in $\mathrm{kW}$ ): $\mathrm{SL}=\{5,9.5,14.8,19.1\}, \mathrm{MTR}=\{1.4,2.8,4.1,5.5\}$, Conv $=\{2,4,6,8\}$ and NLL $=\{1.2,2.4,3.6,4.8,6\}$.
TABLE II

DETAILED DESCRIPTION OF EXPERIMENTAL TEST CONFIGURATIONS

\begin{tabular}{cccccc}
\hline \hline \multirow{2}{*}{ TC } & \multicolumn{4}{c}{ Aggregate load $(\mathbf{k W})$} & \\
\cline { 2 - 5 } & SL & MTR & Conv & NLL & Total \\
\hline TC1 & 14.8 & 5.5 & 6 & 3.6 & 29.9 \\
TC2 & 14.8 & 5.5 & 4 & 6 & 30.3 \\
TC3 & 14.8 & 5.5 & 8 & 2.4 & 30.7 \\
\hline TC4 & 19.1 & 5.5 & 2 & 3.6 & 30.2 \\
\hline TC5 & 19.1 & 5.5 & 4 & 6 & 34.6 \\
\hline TC6 & 19.1 & 5.5 & 6 & 0 & 30.6 \\
TC7 & 19.1 & 0 & 8 & 3.6 & 30.7 \\
TC8 & 19.1 & 0 & 6 & 6 & 31.1 \\
TC9 & 19.1 & 0 & 8 & 0 & 27.1 \\
\hline TC10 & 19.1 & 5.5 & 4 & 4.8 & 33.4 \\
TC11 & 19.1 & 5.5 & 8 & 2.4 & 35.0 \\
\hline TC12 & 19.1 & 5.5 & 4 & 6 & 34.6 \\
TC13 & 9.5 & 5.5 & 2 & 2.4 & 19.4 \\
TC14 & 5 & 5.5 & 6 & 2.4 & 18.9 \\
TC15 & 14.8 & 0 & 2 & 3.6 & 20.4 \\
\hline TC16 & 14.8 & 5.5 & 0 & 0 & 20.3 \\
\hline TC17 & 0 & 5.5 & 6 & 0 & 11.5 \\
\hline TC18 & 14.8 & 0 & 0 & 3.6 & 18.4 \\
\hline \hline
\end{tabular}

Finally, the proposed generic dynamic equivalencing method is compared to a conventional approach, where generic models are developed by applying statistical analysis [7].

\section{A. Evaluation of the Load Disaggregation Procedure}

In Fig. 3, the percentage error, denoted as $P E(\%)$, between the harmonic current vector of the measured aggregate load and the vector sum of the harmonic current spectrum of the corresponding load classes is shown for the different TCs. In most cases, low $P E(\%)$ is observed; higher $P E(\%)$ are mainly observed for the $7^{\text {th }}$ harmonic current.

As discussed in Section II, the loading conditions are determined via disaggregation by finding the minimum difference between the harmonic content of the aggregate load and all possible vector sums generated within HST. In Table III, disaggregation results are evaluated; results are highlighted in color. The green color indicates correct identification of

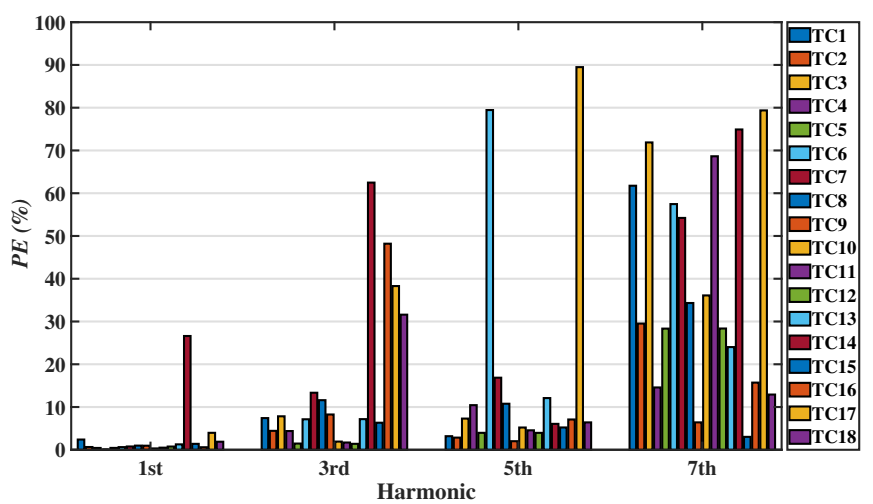

Fig. 3. $P E(\%)$ values between the magnitude of the measured aggregate harmonic current vectors and the magnitude of the harmonic current vectors produced by the corresponding individual connected loads. 
TABLE III

LOAD DISAGGREGATION RESULTS

\begin{tabular}{cccccc}
\hline \hline \multirow{2}{*}{ TC } & \multicolumn{4}{c}{ Aggregate load $(\mathbf{k W})$} & \\
\cline { 2 - 5 } & SL & MTR & Conv & NLL & Total \\
\hline TC1 & 19.1 & 2.8 & 6 & 2.4 & 30.3 \\
TC2 & 19.1 & 1.4 & 6 & 3.6 & 30.1 \\
TC3 & 14.8 & 5.5 & 8 & 2.4 & 30.7 \\
TC4 & 19.1 & 4.1 & 6 & 0 & 29.2 \\
TC5 & 19.1 & 5.5 & 8 & 1.2 & 33.8 \\
TC6 & 19.1 & 5.5 & 6 & 0 & 30.6 \\
TC7 & 19.1 & 0 & 8 & 3.6 & 30.7 \\
TC8 & 14.8 & 5.5 & 8 & 2.4 & 30.7 \\
TC9 & 19.1 & 0 & 8 & 0 & 27.1 \\
TC10 & 19.1 & 5.5 & 8 & 0 & 32.6 \\
TC11 & 19.1 & 5.5 & 8 & 2.4 & 35.0 \\
TC12 & 19.1 & 5.5 & 8 & 1.2 & 33.8 \\
TC13 & 9.5 & 5.5 & 2 & 2.4 & 19.4 \\
TC14 & 14.8 & 1.4 & 0 & 2.4 & 18.6 \\
TC15 & 14.8 & 0 & 2 & 3.6 & 20.4 \\
TC16 & 14.8 & 5.5 & 0 & 0 & 20.3 \\
TC17 & 0 & 5.5 & 4 & 1.2 & 10.7 \\
TC18 & 14.8 & 0 & 0 & 3.6 & 18.4 \\
\hline \hline
\end{tabular}

the loading condition; on the contrary, the red color indicates incorrect identification. A close approximation to the original values (presented in Table II) is represented by the orange color. For example, in TC1, the power of the NLL is $3.6 \mathrm{~kW}$; using the load disaggregation procedure, NLL is estimated equal to $2.4 \mathrm{~kW}$. Results reveal that more than $75 \%$ of the loading conditions for all TCs are adequately identified.

Based on the above analysis, it is shown that the proposed disaggregation scheme can be effectively applied to the harmonic content of the aggregate load to estimate the load composition of the network. Therefore, the fraction of the respective load classes into the total demand can be determined.

\section{B. Generic Dynamic Modelling Results}

In Fig. 4, the curve of WCBCR is depicted by applying k-medoids to the $M_{T D}$ training data sets. Based on the kneepoint criterion, the optimal number of clusters is determined equal to $K=4$. Hence, four representative sets of real and

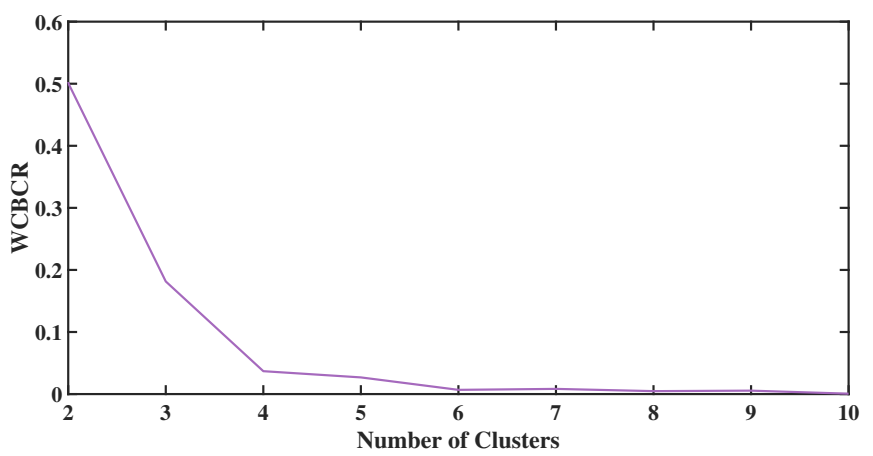

Fig. 4. WCBCR measure assuming different number of clusters.
TABLE IV

REPRESENTATIVE REAL AND REACTIVE POWER MODEL PARAMETERS

\begin{tabular}{cccccccc}
\hline \hline \multirow{2}{*}{ Cluster } & \multicolumn{3}{c}{$\boldsymbol{\theta}_{\boldsymbol{P}, \boldsymbol{k}}^{\prime}$} & & \multicolumn{3}{c}{$\boldsymbol{\theta}_{\boldsymbol{Q}, \boldsymbol{k}}^{\prime}$} \\
\cline { 2 - 4 } \cline { 6 - 8 } & $N_{s, p}$ & $N_{t, p}$ & $T_{y, p}$ & & $N_{s, q}$ & $N_{t, q}$ & $T_{y, q}$ \\
\hline$\# 1$ & 0.634 & 0.655 & 1.775 & & 1.004 & 2.030 & 0.051 \\
$\# 2$ & 0.032 & 0.553 & 156.024 & & 0.635 & 0.456 & 0.872 \\
$\# 3$ & 0.405 & 0.727 & 70.587 & & 0.763 & 0.504 & 1.313 \\
$\# 4$ & 0.075 & 0.440 & 0.546 & & 2.830 & 1.184 & 0.289 \\
\hline \hline
\end{tabular}

reactive power model parameters, i.e., $\boldsymbol{\Theta}_{\boldsymbol{k}}^{\prime}$ (here $k=1, \ldots, 4$ ), are obtained for distinct loading conditions. The indicative sets of model parameters are summarized in Table IV.

To provide an insight in the accuracy of the proposed approach, in Fig. 5, the estimated real and reactive power responses are compared to the corresponding measurements for a random instance of the validation data set. The set of $\Theta_{k}^{\prime}$ employed to derive the generic models corresponds to the one which mostly represents the load composition of the network, using the classification process of subsection II-D. Satisfactory results are obtained for the simulation of both the real and reactive power; here, $\epsilon$ is equal to $0.61 \%$ and $1.02 \%$ for the real and reactive power modelling, respectively.

To validate the robustness of the developed generic models, the cumulative distribution functions (CDF) of $\epsilon(\%)$ resulting from the modelling of the $M_{V D}$ data sets is presented in Fig. 6 . Real power results indicate that in all cases $\epsilon$ is less than $4.5 \%$. Similar results are obtained for reactive power modelling; only four cases are not adequately simulated, i.e., $\epsilon$ is higher than $5 \%$. It is clear that using the proposed generic measurementbased modelling procedure accurate generic models can be obtained. The reactive power responses could have been simulated more accurately by a higher-order dynamic equivalent model, since the oscillatory dynamic behavior presented in some cases cannot be adequately captured using the first-order ERM.

\section{Comparison with other Generic Modelling Approaches}

In order to demonstrate the effectiveness of the proposed approach, the obtained $\epsilon(\%)$ CDF plots are also juxtaposed to the results of generic modelling using statistical analysis
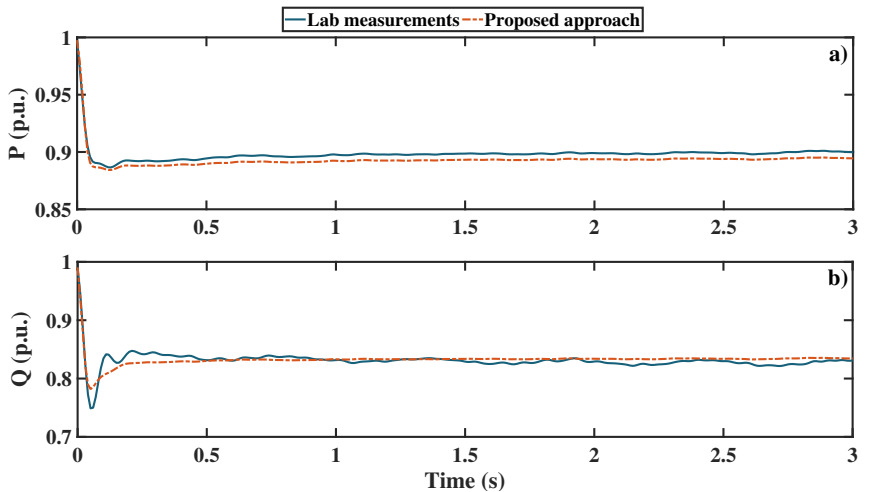

Fig. 5. Comparison of responses for the a) real and b) reactive power. 

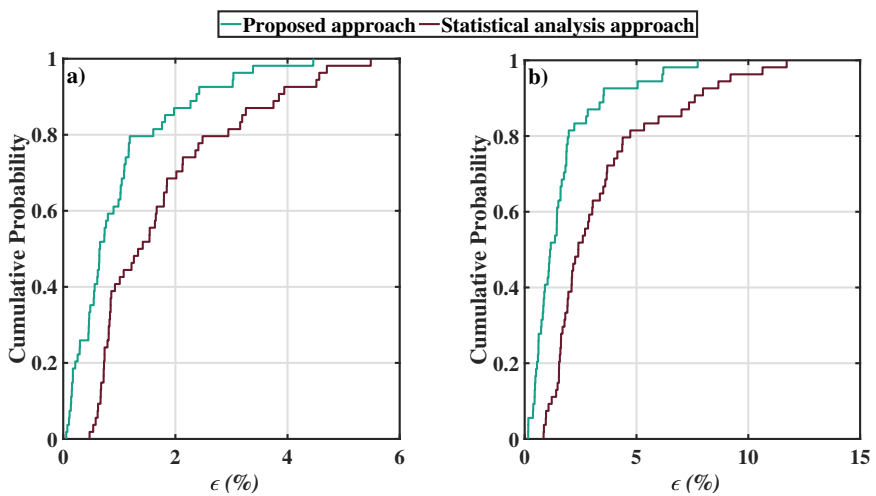

Fig. 6. CDF plots of $\epsilon(\%)$ values for a) real and b) reactive power modelling.

in Fig. 6. In the latter approach, representative sets of model parameters are computed from the available training data sets assuming the mean value of each parameter [7]; the derived generic model parameters are used to simulate the power responses of the $M_{V D}$ validation data sets. It is clear that in all cases lower $\epsilon(\%)$ values are obtained using the proposed generic modelling procedure. Therefore, more accurate generic models are derived compared to those developed by using the statistical analysis.

\section{CONCLUSIONS AND Future WORK}

In this paper, load mix estimates and identified model parameters are forwarded as inputs into a clustering algorithm to derive generic equivalent models. To estimate the load composition of the network, load disaggregation is applied, by analyzing the aggregated harmonic current content under steady-state operation. In addition, the dynamic model parameters are identified by using voltage, real and reactive power disturbance data. Using these inputs, indicative sets of model parameters are acquired for different loading conditions. During the online application, the load mix of the network is continuously determined by decomposing the harmonic content of the aggregated load demand into specific load classes. To simulate unseen disturbances, the set of parameters that mostly represents the estimated load composition of the network is determined using a classification process. The effectiveness of the proposed methodology is validated using experimental measurements obtained in a laboratoryscale distribution system.

Results indicate that using the proposed disaggregation technique the loading condition of specific load classes is adequately identified. Additionally, it is shown that accurate generic measurement-based dynamic equivalent models are obtained. Finally, the proposed approach is compared to a conventional generic modelling procedure, where representative set of model parameters are derived via statistical analysis; in all cases, more accurate results are obtained using the proposed method.

Future work will be devoted to improve the accuracy proposed method. Artificial neural networks techniques will be examined to capture a general relationship between harmonic measurements of the aggregated load and the identified dynamic model parameters. Special emphasis will also be given to validate the performance of the proposed methodology under different network configurations and load classes.

\section{REFERENCES}

[1] Modelling and Aggregation of Loads in Flexible Power Networks. Cigre Working Group C4.605, Technical Report, 2014.

[2] A. Shahsavari, M. Farajollahi, and H. Mohsenian-Rad, "Individual load model parameter estimation in distribution systems using load switching events," IEEE Trans. Power Syst., vol. 34, no. 6, pp. 4652-4664, 2019.

[3] J. V. Milanovic, K. Yamashita, S. Martínez Villanueva, S. Z. Djokic, and L. M. Korunović, "International industry practice on power system load modeling," IEEE Trans. Power Syst., vol. 28, no. 3, pp. 3038-3046, 2013.

[4] A. Arif, Z. Wang, J. Wang, B. Mather, H. Bashualdo, and D. Zhao, "Load modeling - a review," IEEE Trans. Smart Grid, vol. 9, no. 6, pp. 5986-5999, 2018.

[5] V. Knyazkin, C. A. Canizares, and L. H. Soder, "On the parameter estimation and modeling of aggregate power system loads," IEEE Trans. Power Syst., vol. 19, no. 2, pp. 1023-1031, 2004.

[6] Y. Zhu and J. V. Milanović, "Automatic identification of power system load models based on field measurements," IEEE Trans. Power Syst., vol. 33, no. 3, pp. 3162-3171, 2018.

[7] D. P. Stojanović, L. M. Korunović, and J. Milanović, "Dynamic load modelling based on measurements in medium voltage distribution network," Electr. Power Syst. Res., vol. 78, no. 2, pp. 228 - 238, 2008.

[8] H. Renmu, Ma Jin, and D. J. Hill, "Composite load modeling via measurement approach," IEEE Trans. Power Syst., vol. 21, no. 2, pp. 663-672, 2006.

[9] E. O. Kontis, T. A. Papadopoulos, M. H. Syed, E. Guillo-Sansano, G. M. Burt, and G. K. Papagiannis, "Artificial-intelligence method for the derivation of generic aggregated dynamic equivalent models," IEEE Trans. Power Syst., vol. 34, no. 4, pp. 2947-2956, 2019.

[10] S. Mat Zali and J. V. Milanović, "Generic model of active distribution network for large power system stability studies," IEEE Trans. Power Syst., vol. 28, no. 3, pp. 3126-3133, 2013.

[11] K. S. Metallinos, T. A. Papadopoulos, and C. A. Charalambous, "Derivation and evaluation of generic measurement-based dynamic load models," Electr. Power Syst. Res., vol. 140, pp. 193 - 200, 2016.

[12] G. A. Barzegkar-Ntovom, O. Ceylan, T. A. Papadopoulos, and E. Fatih Yetkin, "Generic dynamic load modelling using cluster analysis," in 53rd Int. Univers. Power Eng. Conf. (UPEC), 2018, pp. 1-6.

[13] M. T. Au and J. V. Milanovic, "Development of stochastic aggregate harmonic load model based on field measurements," IEEE Trans. Power Del., vol. 22, no. 1, pp. 323-330, 2007.

[14] D. Karlsson and D. J. Hill, "Modelling and identification of nonlinear dynamic loads in power systems," IEEE Trans. Power Syst., vol. 9, no. 1, pp. 157-166, 1994.

[15] M. T. Au and J. V. Milanovic, "Establishing harmonic distortion level of distribution network based on stochastic aggregate harmonic load models," IEEE Trans. Power Del., vol. 22, no. 2, pp. 1086-1092, 2007.

[16] A. S. Bouhouras, P. A. Gkaidatzis, E. Panagiotou, N. Poulakis, and G. C. Christoforidis, "A NILM algorithm with enhanced disaggregation scheme under harmonic current vectors," Energy and Buildings, vol. 183, pp. 392-407, 2019.

[17] L. Kaufman and P. J. Rousseeuw, Finding groups in data: An introduction to cluster analysis. New York: Wiley, 1990.

[18] G. J. Tsekouras, N. D. Hatziargyriou, and E. N. Dialynas, "Twostage pattern recognition of load curves for classification of electricity customers," IEEE Trans. Power Syst., vol. 22, no. 3, pp. 1120-1128, 2007.

[19] Byoung-Kon Choi, Hsiao-Dong Chiang, Yinhong Li, Hua Li, Yung-Tien Chen, Der-Hua Huang, and M. G. Lauby, "Measurement-based dynamic load models: derivation, comparison, and validation," IEEE Trans. Power Syst., vol. 21, no. 3, pp. 1276-1283, 2006.

[20] A. J. Roscoe, I. F. Abdulhadi, and G. M. Burt, "P and m class phasor measurement unit algorithms using adaptive cascaded filters," IEEE Trans. Power Del., vol. 28, no. 3, pp. 1447-1459, 2013.

[21] A. J. Roscoe, R. Carter, A. Cruden, and G. M. Burt, "Fast-responding measurements of power system harmonics using discrete and fast fourier transforms with low spectral leakage," in IET Conf. Renew. Power Gener, 2011, pp. 1-6. 